\title{
Acoustic Voice Modifications in Individuals with Parkinson Disease Submitted to Deep Brain Stimulation
}

\author{
Aline Juliane Romann ${ }^{1}$ Bárbara Costa Beber ${ }^{2}$ Carla Aparecida Cielo ${ }^{3}$ \\ Carlos Roberto de Mello Rieder ${ }^{1,2,4}$
}

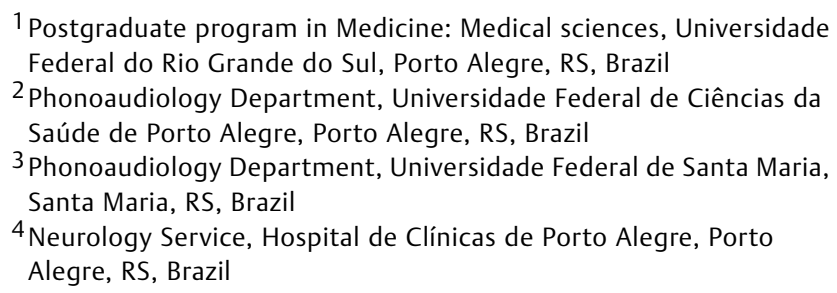

Address for correspondence Carlos R. M. Rieder, Rua Ramiro Barcelos, 2350/ sala 2040; 90035-903 Porto Alegre RS, Brasil (e-mail: carlosrieder@gmail.com).

Int Arch Otorhinolaryngol 2019;23:203-208.

\begin{abstract}
Introduction Subthalamic nucleus deep brain stimulation (STN-DBS) improves motor function in individuals with Parkinson disease (PD). The evidence about the effects of STN-DBS on the voice is still inconclusive.

Objective To verify the effect of STN-DBS on the voice of Brazilian individuals with PD. Methods Sixteen participants were evaluated on the Unified Parkinson Disease Rating Scale-Part III, and by the measurement of the acoustic modifications in on and off conditions of stimulation.

Results The motor symptoms showed significant improvement with STN-DBS on. Regarding the acoustic measures of the voice, only the maximum fundamental frequency (fhi) showed a statistical difference between on- and off-conditions, with

Keywords

- voice

- acoustic analysis

- deep brain stimulation

- parkinson disease reduction in off-condition.

Conclusion Changes in computerized acoustic measures are more valuable when interpreted in conjunction with changes in other measures. The single finding in fhi suggests that DBS-STN increases vocal instability. The interpretation of this result should be done carefully, since it may not be of great value if other measures that also indicate instability are not significantly different.
\end{abstract}

\section{Introduction}

The diagnosis of Parkinson disease (PD) is based on the presence of cardinal motor manifestations, such as tremor, bradykinesia, rigidity, and balance difficulties. ${ }^{1}$ Among many voice and vocal tract dysfunctions observed in these individuals, the most important are hypophonia, reduction of the

(D) Carlos Rieder Roberto de Mello's ORCID is https://orcid.org/ 0000-0003-2950-7211.

received

March 27, 2018

accepted after revision

September 2, 2018

published online

March 1, 2019 maximum phonation times, abnormal movements of the vocal folds, tremor and rigidity in the laryngeal musculature that causes instability in vocal fold vibration, reduction in the velopharyngeal closure, and hypernasal resonance. ${ }^{2-4}$

The current treatments for idiopathic PD rely mainly on the use of pharmacologic agents to improve the motor symptomatology of PD patients, and the most used is levodopa. ${ }^{5}$ Another relatively recent treatment option is a surgical procedure called deep brain stimulation (DBS), and it is used to treat patients with PD when the pharmacological treatment is no
Copyright @ 2019 by Thieme Revinter

Publicações Ltda, Rio de Janeiro, Brazil
License terms

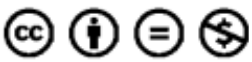

10.1055/s-0038-1675392. ISSN $1809-9777$. 
longer efficient. The subthalamic nucleus (STN) is a frequent target of DBS in PD. ${ }^{5,6}$ It has been proven that DBS suppresses motor symptoms and reduces the total dose of the antiparkinsonian drugs. $^{7-10}$

If on one hand the improvement of the motor function with the use of DBS is reported by several studies, ${ }^{11-13}$ on the other hand the effect of DBS on vocal symptoms is still inconclusive. Most studies have pointed a negative effect of STN-DBS, showing that aspects such as strained voice, breathiness, asthenia, and loudness seem to worsen in the STN-DBS condition. ${ }^{14-16}$ The DBS patients also report more severe symptoms and more interference of these symptoms in their daily experiences than PD patients without DBS. ${ }^{17}$ In contrast, and indicating conflicting results in this field, one study found a positive effect of DBS in the acoustic measures of the voice, as in the case of jitter or frequency perturbation. ${ }^{18}$

It is important to note that different methodological approaches have been used to investigate the voice in this population in terms of DBS condition (pre and postsurgery, onand off-DBS stimulation) and voice assessment tools (subjective assessment of perpetual and psychosocial aspects, objective assessment using acoustic analysis or aerodynamic measures). ${ }^{14,16-22}$ We consider that the investigations of on and off DBS conditions is the best choice to evaluate the effects of the stimulation per se, since the investigation of pre and postsurgery conditions is biased by the effects of the surgical procedure, such as possible brain lesions caused by the surgery. Furthermore, different voice assessment methods also provide different information. Objective measures are very relevant for scientific purposes because they offer more reliable objective data and, in this case, the acoustic analysis plays this role.

In the present study, we have investigated the effect of STNDBS on the voice of PD patients. The strengths of our study are the addition of information about the Brazilian population; evaluation of on- and off-DBS conditions; the usage of the Multi Dimensional Voice Program Advanced (MDVPA) (KayPENTAX, Lincoln Park, NJ, USA) software, which analyzes an extensive number of acoustic variables, and the assessment of the participants in a more naturalistic situation that maintains their regular pharmacological treatment.

\section{Methods}

\section{Participants}

Sixteen Brazilian patients with PD who had undergone bilateral STN-DBS were selected from the Movement Disorder Clinic at a public hospital in Porto Alegre and were included in the present study. Participants with a poor response to the procedure, presence of another neurological condition (such as stroke or dementia), those who reported auditory impairment, and those who were not native speakers of Brazilian Portuguese were not included. The study was approved by the local ethics committee (n. 10.0508) and all of the participants signed a written informed consent.

\section{Instruments and Procedures}

Clinical and demographic data were obtained from the records of the patients to describe the sample. The assess- ment team consisted of one movement disorder neurologist, and of three speech and language therapists.

The motor function of the patients was assessed using the Unified Parkinson Disease Rating Scale (UPDRS) part III (maximum score of 108 points). Sample voices were recorded using a unidirectional Philips SBC MD 195/00 microphone, (Philips Healthcare, Amsterdam, Netherlands) fixed on a pedestal and positioned at a $45^{\circ}$ angle from the mouth of the participants. The participants were seated, due to the difficulty of standing, and requested to emit a sustained sound of vowel /a/ at their habitual loudness and pitch, maintaining a distance of $4 \mathrm{~cm}$ between the microphone and the mouth. The duration of all recordings was standardized in one-second segments; at least one second of the initial part and one second of the final part were cut. The duration of one second was defined based on the average duration of the emissions of the participants, which are short in patients with $\mathrm{PD}$, since a reduction in maximum phonation times is a consequence of the disease.

Recordings were analyzed using the MDVPA software. The MDVPA analyzes several vocal measures, which are the following:

- Frequency measures: fundamental frequency (f0); maximum f0 (fhi); minimum f0 (flo); standard deviation of f0 (std);

- Frequency perturbation measures: relative average perturbation (rap); percentage jitter (jitt); absolute jitter (jita); smoothed pitch perturbation quotient (sppq); pitch perturbation quotient (ppq); coefficient of variation of f0 (vf0);

- Amplitude perturbation measures: shimmer in $\mathrm{dB}$ (shdb); shimmer percentage (shim); coefficient of variation of amplitude (vam); amplitude perturbation quotient (apq); smoothed coefficient of variation of amplitude (sapq);

- Noise measures: noise-harmonic ratio (nhr); smooth phonation index (spi); voice turbulence index (vti);

- Voice breaking measures: number of voice breaks (nvb); degree of voice breaks (dvb);

- Mute or unvoiced segments measures: degree of unvoiced segment (duv); number of unvoiced segment (nuv);

- Sub-harmonic components measures: numbers of subharmonic segments (nsh);

- Tremor measures: f0-tremor intensity index (ftri); amplitude tremor intensity index (atri); f0-tremor frequency (fftr); amplitude tremor frequency (fatr).

The UPDRS-III and the voice recordings were performed on the same day with patients on their usual antiparkinsonian medication, in the following conditions: 1) on-stimulation: the patients were evaluated with the DBS turned on and adjusted for the best symptom control by each patient (baseline); 2) off-stimulation: the DBS was turned off and the assessments were performed after 60 minutes or until the patient could not tolerate the symptoms. At the end of the off-stimulation evaluation, the DBS was turned on again.

\section{Statistical Analysis}

Statistical analyses were performed using the SPSS Statistics for Windows, Version 21.0 (IBM Corp, Armonk, NY, USA) with a significance level of $5 \%(p \leq 0.05)$. The continuous variables 
Table 1 Baseline characteristics of the participants

\begin{tabular}{|l|l|}
\hline Variables & Distribution \\
\hline Gender-Total/Male $(\mathrm{n}, \%)$ & $16 / 12(75)$ \\
\hline Age (mean \pm SD) & $57.25 \pm 14.08$ \\
\hline Education (mean $\pm \mathrm{SD})$ & $12.06 \pm 4.20$ \\
\hline Time of disease - years (mean $\pm \mathrm{SD})$ & $12.31 \pm 4.02$ \\
\hline Time after surgery-months (mean \pm SD) & $6.75 \pm 8.62$ \\
\hline Frequency of DBS-Hz (mean \pm SD) & $156.25 \pm 29.92$ \\
\hline Amplitude of DBS, left-V (mean \pm SD) & $2.98 \pm 0.56$ \\
\hline Amplitude of DBS, right-V (mean \pm SD) & $2.94 \pm 0.59$ \\
\hline Pulse width of DBS, left- $\mu$ s (mean \pm SD) & $84.38 \pm 19.65$ \\
\hline Pulse width of DBS, right- $\mu$ s (mean \pm SD) & $90.00 \pm 19.97$ \\
\hline
\end{tabular}

Abbreviations: DBS, deep brain stimulation; $\mathrm{Hz}$, Hertz; $\mu$ s, microsecond; SD, standard deviation; $V$, Volt.

were reported as mean and standard deviation (SD). The categorical variables were described by absolute and relative frequencies. A generalized estimating equation (GEE) model was used to compare the acoustic measures of the voice and the UPDRS-III performances between on- and off-stimulation conditions. Given that gender may implicate in differences in glottal source and voice, and because we did not have an adequate sample size to do a separate analysis, it was used as a covariate in the analyses.

\section{Results}

The baseline characteristics of the 16 participants included in the present study are described in -Table 1.

- Table 2 presents the comparisons of UPDRS-III and all the acoustic measures between on- and off-stimulation conditions. We have found a significant difference in UPDRS-III indicating better motor performance when patients were assessed with DBS turned on. The only acoustic measure that presented a significant difference was the fhi, which was significantly higher in the on-DBS condition.

\section{Discussion}

The aim of the present study was to verify the effect of STNDBS on the voices of individuals with PD by measuring the acoustic modifications between on- and off-stimulation conditions. In our sample, the motor symptoms showed a significant improvement with STN-DBS on (-Table 2). The improvement of motor patterns in the presence of DBS stimulation is already a consensus, ${ }^{23,24}$ and that is the reason why we use it as a parameter of the impact of STN-DBS on parkinsonian symptoms. In the vocal analysis, only one acoustic measure of the voice (fhi) showed a statistical difference between on- and off-DBS conditions (-Table 2 ).

The UPDRS-III assesses the motor aspects and focuses more on the limbs, but also on the speech and on the face, while the computerized acoustic analysis assesses glottal source measures, which rely on voluntary and involuntary laryngeal movements. The fact that the studied sample presented an improvement in motor symptoms measured by the UPDRS-III and not in the acoustic measures suggests that the motor control of the voice does not occur in the same way that the motor control of other body parts, and also that DBS affects the motor control of different body parts differently.

The fhi is the highest fundamental frequency reached in all periods extracted from the acoustic wave. ${ }^{25}$ High fhi values suggest a greater variability in the voice substation, and consequently, a greater vocal instability. ${ }^{26,27}$ It has already been demonstrated that patients with PD present higher values of fhi as a consequence of their vocal instability. ${ }^{28}$ Based on this, we could infer that, in our study, DBS increased the fhi and then the vocal instability. However, vocal changes detected by the computerized acoustic measures have more value when interpreted in conjunction with changes in other similar measures. In the present case, the finding regarding the fhi may not be of great value if other measures that also indicate instability are not significantly different. In the present case, our results suggest that STNDBS increases vocal instability, but if we consider that other measures of instability were not influenced by DBS, we can assume that STN-DBS did not affect the acoustic vocal measures in the studied sample.

Studies that have also compared the effects of the neurostimulator between on- and off-conditions found no difference in the acoustic measurements. In a study ${ }^{29}$ that performed acoustic measurements using the PRAAT software (Paul Boersma and David Weenik, University of Amsterdam, Amsterdam, Netherlands) and perceptual voice analysis with 38 patients with idiopathic PD and chronic bilateral STN-DBS, each patient was tested in the stimulation conditions on and off, and off medication. The researchers found no significant change in the acoustic analysis of the voice. However, there was a trend of improvement of voice quality and prosody in the STN-DBS on condition. Other researchers ${ }^{30}$ investigated the effects of bilateral STN-DBS on the phonation of PD patients in three drug-free conditions: stimulation off, with clinically optimized stimulation parameters, and subthreshold overstimulation. The acoustic analysis performed through the PRAAT program showed no significant changes in the perturbation measures studied (jitt, shim, and nhr) for the aforementioned conditions. Another study ${ }^{31}$ evaluated the acoustic aspects of the voice, using the software package Computerized Speech Lab Multi Dimensional Voice Program (MDVP) - Kay-Elemetrics Model 430 (Pentax Lifecare, Tokyo, Japan), in 19 patients with PD with bilateral STN-DBS, in on- and off-stimulation conditions, and under medication. They also did not find a significant alteration.

Therefore, we have different hypotheses to explain the results presented in this study:

- First, many aspects may influence the voice outcomes in people with PD and DBS, and because they were not explored or controlled in the present study, their effects were not detected. Some studies suggest that the negative 
Table 2 Comparisons of acoustic measures of the voice between the different frequencies of SNT-DBS

\begin{tabular}{|c|c|c|c|}
\hline & DBS ON & DBS OFF & \\
\hline & Mean \pm SE & Mean \pm SE & p-value \\
\hline UPDRS-III & $24.19 \pm 2.68$ & $49.25 \pm 6.84$ & $0.000^{*}$ \\
\hline \multicolumn{4}{|c|}{ Frequency measures $^{\mathrm{a}}$} \\
\hline $\mathrm{f}_{0}(\mathrm{~Hz})$ & $154.66 \pm 8.95$ & $152.61 \pm 10.63$ & 0.522 \\
\hline fhi $(\mathrm{Hz})$ & $220.23 \pm 25.81$ & $170.61 \pm 10.46$ & $0.038^{*}$ \\
\hline flo $(\mathrm{Hz})$ & $129.76 \pm 9.97$ & $134.22 \pm 14.48$ & 0.689 \\
\hline STD $(\mathrm{Hz})$ & $9.45 \pm 3.86$ & $3.63 \pm 0.81$ & 0.129 \\
\hline \multicolumn{4}{|c|}{ Frequency perturbation measures $^{a}$} \\
\hline jitta (us) & $199.94 \pm 66.93$ & $94.95 \pm 28.42$ & 0.090 \\
\hline Jitt (\%) & $2.63 \pm 0.81$ & $1.27 \pm 0.31$ & 0.073 \\
\hline RAP (\%) & $1.58 \pm 0.50$ & $0.72 \pm 0.17$ & 0.071 \\
\hline PPQ (\%) & $1.58 \pm 0.47$ & $0.81 \pm 0.21$ & 0.085 \\
\hline sPPQ (\%) & $3.09 \pm 1.14$ & $1.39 \pm 0.37$ & 0.132 \\
\hline $\mathrm{vf}_{0}(\%)$ & $6.44 \pm 2.58$ & $2.64 \pm 0.69$ & 0.136 \\
\hline \multicolumn{4}{|c|}{ Amplitude perturbation measures $^{a}$} \\
\hline ShdB $(\mathrm{dB})$ & $0.67 \pm 0.17$ & $0.52 \pm 0.15$ & 0.364 \\
\hline Shim (\%) & $6.97 \pm 1.71$ & $5.72 \pm 1.63$ & 0.489 \\
\hline APQ (\%) & $5.19 \pm 1.16$ & $4.42 \pm 1.26$ & 0.532 \\
\hline SAPQ (\%) & $6.78 \pm 1.40$ & $6.31 \pm 1.75$ & 0.761 \\
\hline vAm (\%) & $14.29 \pm 3.20$ & $10.73 \pm 2.72$ & 0.169 \\
\hline \multicolumn{4}{|c|}{ Noise measures $^{a}$} \\
\hline NHR & $0.25 \pm 0.04$ & $0.19 \pm 0.03$ & 0.123 \\
\hline VTI & $0.05 \pm 0.00$ & $0.05 \pm 0.00$ & 0.530 \\
\hline SPI & $9.34 \pm 0.95$ & $9.57 \pm 0.81$ & 0.819 \\
\hline \multicolumn{4}{|c|}{ Voice break measures $^{a}$} \\
\hline DVB (\%) & $1.61 \pm 1.40$ & $0.74 \pm 0.71$ & 0.576 \\
\hline NVB & $0.13 \pm 0.09$ & $0.07 \pm 0.07$ & 0.580 \\
\hline \multicolumn{4}{|c|}{ Subharmonic components measures $^{\mathrm{a}}$} \\
\hline DSH (\%) & $1.60 \pm 0.86$ & $0.14 \pm 0.14$ & 0.100 \\
\hline $\mathrm{NSH}$ & $1.33 \pm 0.72$ & $0.14 \pm 0.14$ & 0.109 \\
\hline \multicolumn{4}{|c|}{ Mute or Unvoiced Segments Measures ${ }^{a}$} \\
\hline DUV (\%) & $13.83 \pm 5.78$ & $8.63 \pm 5.33$ & 0.243 \\
\hline NUV & $13.53 \pm 5.61$ & $8.14 \pm 5.03$ & 0.183 \\
\hline \multicolumn{4}{|c|}{ Tremor measures $^{a}$} \\
\hline $\mathrm{Fftr}(\mathrm{Hz})$ & $4.64 \pm 0.82$ & $4.26 \pm 0.88$ & 0.762 \\
\hline Fatr $(\mathrm{Hz})$ & $5.42 \pm 0.73$ & $5.97 \pm 1.28$ & 0.614 \\
\hline FTRI (\%) & $0.90 \pm 0.19$ & $0.79 \pm 0.17$ & 0.539 \\
\hline ATRI (\%) & $5.80 \pm 1.87$ & $2.87 \pm 0.90$ & 0.063 \\
\hline
\end{tabular}

Abbreviations: APQ, amplitude perturbation quotient; ATRI, amplitude tremor intensity rate; DSH, degree of sub-harmonics; DUV, degree of unvoiced segments; DVB, degree of voice breaks; $\mathrm{f0}$, average fundamental frequency; Fatr, tremor frequency amplitude; fhi, maximum $\mathrm{f}_{0}$; $\mathrm{Fftr}$, tremor frequency; flo, maximum $\mathrm{f}_{0}$; FTRI, tremor intensity rate; jitta, absolute jitter; jitt, jitter percentage; NHR, noise-to-harmonics Ratio; nsh, number of sub-harmonics; NUV, number of unvoiced segments; NVB, number of voice breaks; PPQ, pitch perturbation quotient; RAP, relative measure of the pitch disturbance; SAPQ, smoothed amplitude perturbation quotient; SE, standard error; ShdB, shimmer in dB; Shim, percentage shimmer; SPI, smoothed phonation index; SPPQ, smoothed pitch perturbation quotient; STD, standard deviation; UPDRS-III, Unified Parkinson Disease Rating Scale part III, vAm, amplitude variation; Vf0, $\mathrm{f}_{0}$ variation; VTI, voice turbulence index.

${ }^{a}$ Gender was used as a covariate; ${ }^{*} p \leq 0.05$. 
effect observed in the voice of people with PD and DBS may be due to aging, to PD itself, and to corticobulbar effects. ${ }^{16}$ Other studies have suggested that DBS parameters such as the voltage and the frequency of stimulation might influence the voice in different ways. ${ }^{32}$ The vocal apparatus and the voice may differ according to race, gender, and general physical characteristics. ${ }^{33,34}$ However, nothing is known about how these features interact with the effect of DBS on the voices of people with PD.

- Second, it might be true that STN-DBS does not affect the acoustic measures of voice. The pyramidal and extrapyramidal circuitry of different motor areas is differently organized in terms of somatotopy in cortical brain areas and in the STN. It is possible that STN-DBS affects the motor control of the limbs, for example, but does not affect the motor control of the larynx.

- Third, computerized acoustic analysis may not be able by itself to detect vocal changes of a glottic source in this specific population.

\section{Conclusions}

In conclusion, the single finding in fhi suggests that DBS-STN increases vocal instability. But because this change was not followed by changes in other measures of vocal instability, our results suggest no important effect of STN-DBS in vocal acoustic measures of Brazilian individuals with PD. Future studies should analyze the different voice measures by expanding the sample as well as including other instruments of vocal evaluation that can add information, since the literature does not present conclusive results.

\section{References}

1 Braak H, Del Tredici K, Rüb U, de Vos RA, Jansen Steur EN, Braak E. Staging of brain pathology related to sporadic Parkinson's disease. Neurobiol Aging 2003;24(02):197-211

2 Logemann JA, Fisher HB, Boshes B, Blonsky ER. Frequency and cooccurrence of vocal tract dysfunctions in the speech of a large sample of Parkinson patients. J Speech Hear Disord 1978;43(01): 47-57

3 Darley FL, Aronson AE, Brown JR. Differential diagnostic patterns of dysarthria. J Speech Hear Res 1969;12(02):246-269

4 Yorkston KM, Beukelm DR, Bell KR. Clinical management of dysarthric speakers. 1st ed. Boston: College-Hill; 1988

5 Pires AO, Teixeira FG, Mendes-Pinheiro B, Serra SC, Sousa N, Salgado AJ. Old and new challenges in Parkinson's disease therapeutics. Prog Neurobiol 2017;156:69-89

6 Machado A, Fernandez HH, Deogaonkar M. Deep brain stimulation: what can patients expect from it? Cleve Clin J Med 2012;79 (02):113-120

7 Weaver FM, Follett KA, Stern M, et al; CSP 468 Study Group. Randomized trial of deep brain stimulation for Parkinson disease: thirty-six-month outcomes. Neurology 2012;79(01):55-65

8 Fasano A, Daniele A, Albanese A. Treatment of motor and nonmotor features of Parkinson's disease with deep brain stimulation. Lancet Neurol 2012;11(05):429-442

9 Hammer MJ, Barlow SM, Lyons KE, Pahwa R. Subthalamic nucleus deep brain stimulation changes speech respiratory and laryngeal control in Parkinson's disease. J Neurol 2010;257(10):1692-1702
10 Aström M, Tripoliti E, Hariz MI, et al. Patient-specific model-based investigation of speech intelligibility and movement during deep brain stimulation. Stereotact Funct Neurosurg 2010;88(04):224-233

11 Wolfe VI, Garvin JS, Bacon M, Waldrop W. Speech changes in Parkinson's disease during treatment with L-dopa. J Commun Disord 1975;8(03):271-279

12 Lee VS, Zhou XP, Rahn DA, Wang EQ, Jiang JJ. Perturbation and nonlinear dynamic analysis of acoustic phonatory signal in Parkinsonian patients receiving deep brain stimulation. J Commun Disord 2008;41(06):485-500

13 Zangaglia R, Pacchetti C, Pasotti C, et al. Deep brain stimulation and cognitive functions in Parkinson's disease: A three-year controlled study. Mov Disord 2009;24(11):1621-1628

14 Lundgren S, Saeys T, Karlsson F, et al. Deep brain stimulation of caudal zona incerta and subthalamic nucleus in patients with Parkinson's disease: effects on voice intensity. Parkinsons Dis 2011;2011:658956

15 Tanaka Y, Tsuboi T, Watanabe H, et al. Voice features of Parkinson's disease patients with subthalamic nucleus deep brain stimulation. J Neurol 2015;262(05):1173-1181

16 Tsuboi T, Watanabe H, Tanaka Y, et al. Distinct phenotypes of speech and voice disorders in Parkinson's disease after subthalamic nucleus deep brain stimulation. J Neurol Neurosurg Psychiatry 2015;86(08):856-864

17 Wertheimer J, Gottuso AY, Nuno M, et al. The impact of STN deep brain stimulation on speech in individuals with Parkinson's disease: the patient's perspective. Parkinsonism Relat Disord 2014;20(10):1065-1070

18 Mate MA, Cobeta I, Jiménez-Jiménez FJ, Figueiras R. Digital voice analysis in patients with advanced Parkinson's disease undergoing deep brain stimulation therapy. J Voice 2012;26(04):496-501

19 Frost E, Tripoliti E, Hariz MI, Pring T, Limousin P. Self-perception of speech changes in patients with Parkinson's disease following deep brain stimulation of the subthalamic nucleus. Int J SpeechLanguage Pathol 2010;12(05):399-404

20 Tsuboi T, Watanabe H, Tanaka Y, et al. Characteristic laryngoscopic findings in Parkinson's disease patients after subthalamic nucleus deep brain stimulation and its correlation with voice disorder. J Neural Transm (Vienna) 2015;122(12):1663-1672

21 Van Lancker Sidtis D, Rogers T, Godier V, Tagliati M, Sidtis JJ. Voice and fluency changes as a function of speech task and deep brain stimulation. J Speech Lang Hear Res 2010;53(05):1167-1177

22 Zhou XP, Lee VS, Wang EQ, Jiang JJ. Evaluation of the effects of deep brain stimulation of the subthalamic nucleus and levodopa treatment on parkinsonian voice using perturbation, nonlinear dynamic, and perceptual analysis. Folia Phoniatr Logop 2009;61 (04):189-199

23 Fox SH, Katzenschlager R, Lim SY, et al. The movement disorder society evidence-based medicine review update: treatments for the motor symptoms of Parkinson's disease. Mov Disord 2011;26 (Suppl 3):S2-S41

24 Moldovan AS, Groiss SJ, Elben S, Südmeyer M, Schnitzler A, Wojtecki L. The treatment of Parkinson's disease with deep brain stimulation: current issues. Neural Regen Res 2015;10(07):1018-1022

25 González J, Cervera T, Miralles JL. Acoustic voice analysis: reliability of a set of multi-dimensional parameters. Acta Otorrinolaringol Esp 2002;53(04):256-268

26 Beber BC, Cielo CA. Acoustic measurements of the glottal source of normal male voices. Pro Fono 2010;22(03):299-304

27 Colman Machado de Machado F, Cielo CA, Lessa MM, Barbosa LHF. Vocal characteristics of elderly women engaged in aerobics in private institutions of Salvador, Bahia. J Voice 2016;30(01):127.e9-127.e19

28 Ferreira FV, Cielo CA, Trevisan ME. Medidas vocais acústicas na doença de Parkinson: estudo de casos. Rev CEFAC 2010;12(05): 889-898

29 Skodda S, Grönheit W, Schlegel U, Südmeyer M, Schnitzler A, Wojtecki L. Effect of subthalamic stimulation on voice and speech 
in Parkinson's disease: for the better or worse? Front Neurol 2014; 4:218

30 Valálik I, Smehák G, Bognár L, Csókay A. Voice acoustic changes during bilateral subthalamic stimulation in patients with Parkinson's disease. Clin Neurol Neurosurg 2011;113(03):188-195

31 Klostermann F, Ehlen F, Vesper J, et al. Effects of subthalamic deep brain stimulation on dysarthrophonia in Parkinson's disease. J Neurol Neurosurg Psychiatry 2008;79(05):522-529
32 Moreau C, Pennel-Ployart O, Pinto S, et al. Modulation of dysarthropneumophonia by low-frequency STN DBS in advanced Parkinson's disease. Mov Disord 2011;26(04):659-663

33 Inamoto Y, Saitoh E, Okada S, et al. Anatomy of the larynx and pharynx: effects of age, gender and height revealed by multidetector computed tomography. J Oral Rehabil 2015;42(09):670-677

34 Walton JH, Orlikoff RF. Speaker race identification from acoustic cues in the vocal signal. J Speech Hear Res 1994;37(04):738-745 